\title{
Zhuang Opera --- A study of Chinese Minority Arts in Guangxi Province, China
}

\author{
Zhang Jian', Arsenio Nicolas ${ }^{2}$ \\ College of music, Mahasarakham University
}

Article History: Received: 10 November 2020; Revised 12 January 2021 Accepted: 27 January 2021; Published online: 5 April 2021

\begin{abstract}
This article is a study of Zhuang Opera in the Guangxi Province in China. There are three general genres of Zhuang Opera, the analysis of selected musical pieces of these four types focuses on the music structure of Pingban melody, and the rhyme of the song texts --- Yaojiaoyun. This study is a pioneering work on the Zhuang Opera's musical form as there is currently no published work on the music of the opera and its association with related music categories. Except for very few research collections on national opera, most studies mainly focus on the history, literature, and folklore of traditional Zhuang Opera. In general, this study utilized new research methodologies producing different results from previous research, while at the same time, confirming earlier studies on the music and performance styles of the opera.
\end{abstract}

Keywords: Chinese opera, Zhuang music, Music transmission, Minority art

\section{Introduction}

Zhuang Opera has high historical, literary, and artistic values while condensing within itself rich aesthetic connotations and profound cultural connotations. In the long and tortuous life course of the Zhuang Opera, the literati and entertainers have carefully crafted it, introducing brilliant instrumental music, vocal melodies, and performing styles. Whether at the peak or low tide of development, the Zhuang Opera vividly recorded the social life of the ancient Zhuang people in China and kept its aesthetic characteristics, which profoundly affected the formation and development of other Zhuang arts. In addition, Zhuang Opera has always been respected as a model of operatic art for ethnic minorities. Zhuang Opera is the

1 Ph.D. Candidate, Collage of Music, Mahasarakham University, Thailand

2 Advisor, Collage of Music, Mahasarakham University, Thailand 
essence of Chinese Zhuang culture and the precious spiritual wealth of humanity (Li Fuqiang, 1999).

As the ethnic minority with the largest population in China, there is still a lack of research on the national Zhuang arts. Under the impact of multiculturalism, all kinds of fashionstimulating entertainment and leisure-time activity are overwhelming, especially for young people who prefer film and television, the internet, and other entertainment methods that allow them to vent their emotions. At the same time, the talent training and performance practice of Zhuang Opera require not only low investment costs but also long-term training. Therefore, although the current situation of Zhuang Opera has become better, the various crises it faces still exist (Liao Mingjun, 2008).

In recent years, there has been an increasing amount of academic research on Zhuang Opera, but most of the previous studies are on ethnology and folklore from the perspectives of its historical origin, transmission, and its development of vocal and stage performance arts. There are relatively few studies focusing on the contemporary development of Zhuang Opera, and there are no monographs or research to study the music characteristics of Zhuang Opera.

The research objectives of this study have the following three points: 1) to investigate the characteristics of Zhuang Opera, including the the period of time from the original to the present, vocal music, instrumental music, the earliest form of Zhuang Opera “Taipingchun”, as well as its development process; 2) to analyze the selected music pieces of Zhuang Opera. In this part, the original music forms were analyzed from the perspective of ethnomusicology and musicology, as well as the music characteristics from the aspect of music analysis, including the relationship of the melodies, and the rhymes.

This research expands the scope of conceptual research, and is also an extension of the academic circle of art. At the same time, it also provides references for other researchers in research models and conceptual applications.

\section{Literature Review}

Zhuang Opera is a form of performing art among Zhuang people, it exudes a unique charm in their folk practices. Since the beginning of the 1950s, some scholars have studied the art of Zhuang Opera from different angles. As an essential part of ethnic minorities' opera, it has received many scholars' attention and has produced many research results.

\subsection{General research on Zhuang Opera in Guangxi Province}

Wei Wei and Xiang Fan (1990) wrote the first monograph on the art of Zhuang Opera. This book explores its detailed and systematic elaboration from the aspects of its repertoire, music, performance, stage art, and revitalization, which provided a useful reference for future 
generations to understand and study its art.

The "Chinese Ethnic Minority Opera Research Essays," edited by Fang Hechun (2007), is the first thesis on Chinese operas, which covers 14 operas, the Zhuang Operas' distribution, historical origins, and artistic skills.

\subsection{Research on the music characteristics of zhuang opera}

Lu Guomin (2008) wrote "The Development of Zhuang Opera and the Formation of Music" in which they mainly discussed the formation of Yunnan Zhuang Opera, its music structure, and vocal characteristics.

Yang Danhua (2013) analyzed traditional and modern singing methods from two different eras and then obtained the folk singing, chanting, and physical styles used in Zhuang Opera singing. The author points out that one must pay attention to the suitable combination of singing form and content.

There are also some western scholars who have thought that although cultural cadres were restored after the Cultural Revolution, and some aspects of traditional culture are mentioned in school curricula, these efforts have done little to stem the waning of traditional culture among the Zhuang (Widman and John Harvey, 2019).

Bodomo and Adams pointed this out in "Strategies for the Documentation and Revitalization of the Zhuang Language of Southwestern China" (Bodomo and Adams, 2007).

Becker and Judith (2004) discussed Zhuang Opera from three aspects: background, music structure, and singing language, which have influencing factors and express the style of the times. The viewpoint described how to develop a heritage and return to its original artistic essence.

\subsection{RESEARCH ON ZHUANG OPERA REPERTOIRE}

Huang Shouheng (2015) studied the pedigree of his teacher's biography by sorting out the content of "Tai Ping Chun," the founding work of North Zhuang Opera, and by tracing its origins. His other thesis “Zhuang Zi Ancient Books 'Tai Ping Chun' language studies” detailed an analysis of its phonetic system, vocabulary and rhyme rhetoric.

\section{Research Methodology}

This research is conducted qualitative methodology, and the writing method used is descriptive analysis. In this research process, the researchers collected information in two ways: field work and literature review. First of all, in the field survey, the researchers interviewed some artists of Zhuang Opera, and members of the Zhuang Opera troupe. The content of the 
interview mainly includes the development process of Zhuang Opera, how the transmission works. Secondly, according to the collected documents and data, information on the development and the music characteristics of of Zhuang Opera was analyzed.

\section{The characteristics of Zhuang Opera}

\subsection{The classification of Zhuang Opera}

The Zhuang Opera is the traditional opera in the Zhuang area. It is an artistic performance form based on the Zhuang's rich folk tales, music and dance, and customs. After the People's Republic of China was founded (1949), it was named "Zhuang Opera" (Wei Wei and Xiang Fan, 1990).

There are many different views on and approaches to the classification of Zhuang Opera. Many scholars have proposed different classification methods from different angles. Some propose that Guangxi Zhuang Operas include Tianlong Zhuang Opera, Jingde Zhuang Opera, and Hanlong Zhuang Opera. Others divide it into Longlin Zhuang Opera, Tianlin Zhuang Opera, Jingde Puppet Zhuang Opera, Shide Opera, and Ma’ai Zhuang Opera (Huang Youxia, 2008).

These differences are reflected in the natural geographical environment, cultural environment, the characteristics of the local dialect, and differences in the vocal melody of the Zhuang Opera. Given the different types of classification mentioned above, this research will analyze Zhuang Opera according to the following classification: North Zhuang Opera, South Zhuang Opera, and Shigong Zhuang Opera (Zhang Jiannian, 1987).

Table 1 The classification of Zhuang Opera

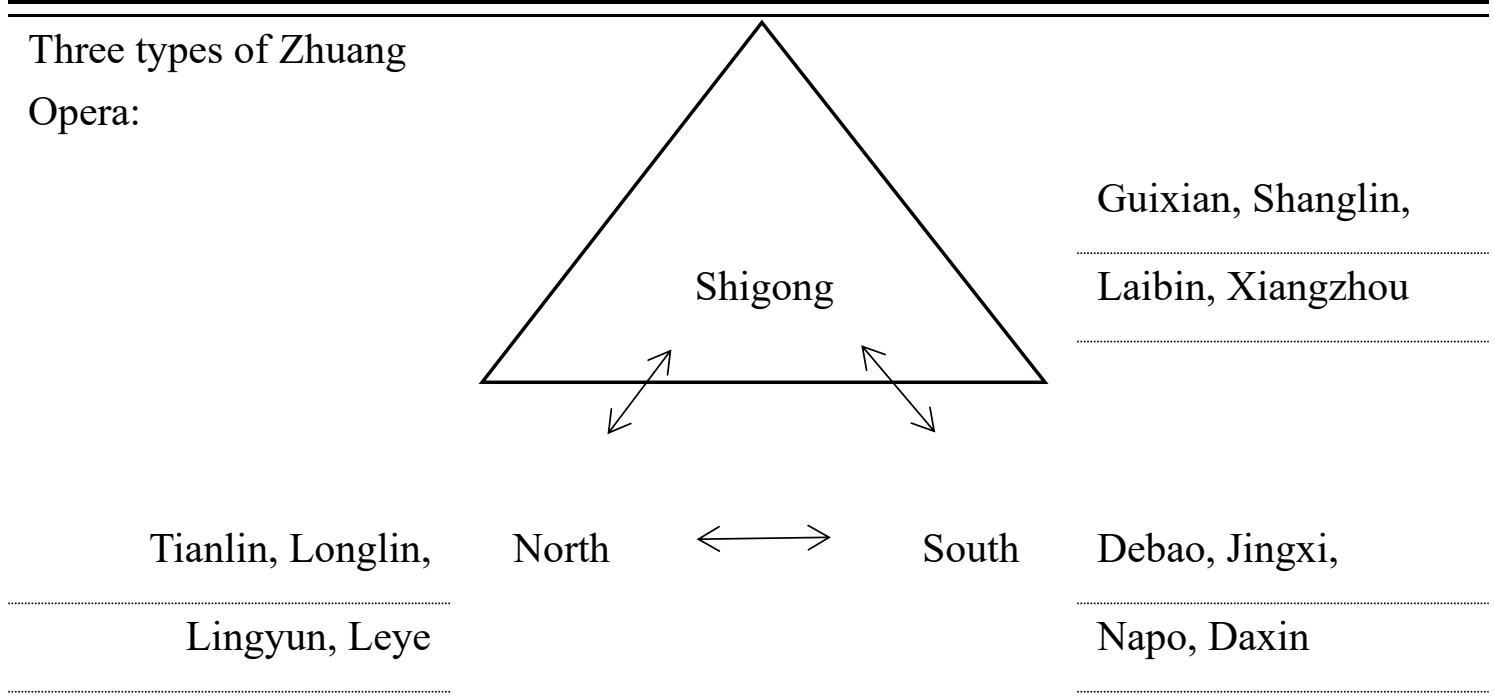

Note: This classification method will be used in this study 


\subsection{The development process of Zhuang Opera}

The origin of the North Zhuang Opera can be traced back to the late Ming and early Qing dynasties. At that time, the Bayin had been introduced to Jiuzhou Town in Tianlin County, and other places. The so-called Bayin refers to playing with eight kinds of instruments, including the suona, dizi, erhu, and pipa; as well as four percussion instruments described as a small drum, small cymbal, small gong, and bronze bell (Han Wanghong, 2017).

In the Song dynasty, after the formation of traditional Chinese opera, Bayin continued to absorb fresh elements from it to enrich its artistic form and increase the performance form, which appeared as a new performance style accompanied by instrumental performance and singing, named “Bayin Sitting-singing” (八音坐唱). As the ancient book “Lingwaidaida” recorded, "Guangxi people can have fun together, sacrifice, marry, have funerals, with all participants having fun. Even while farming, they will sing-song at the same time" (Tian Qing, 2017).

In the late years of the Guangxu period of the Qing Dynasty (1871-1908), Huang Fuxiang, an artist of the Zhuang Opera, he changed the imprisonment of traditional thinking by actively mobilizing women to participate in acting, innovating the costumes, making the roles of men and women fully equal, and the first time opened up Zhuang Opera male and female acting roles to be on the same stage together (Fang Hechun, 2007).

The Zhuang nationality's representative stringed instrument is the maguhu (马骨胡), which is two-stringed, and uses a hollowed-out horse femur for a resonator. In both academic and popular literature, the maguhu is commonly referred to as a uniquely Zhuang instrument played in instrumental ensembles and in the Zhuang Opera (Widman and John Harvey, 2019).

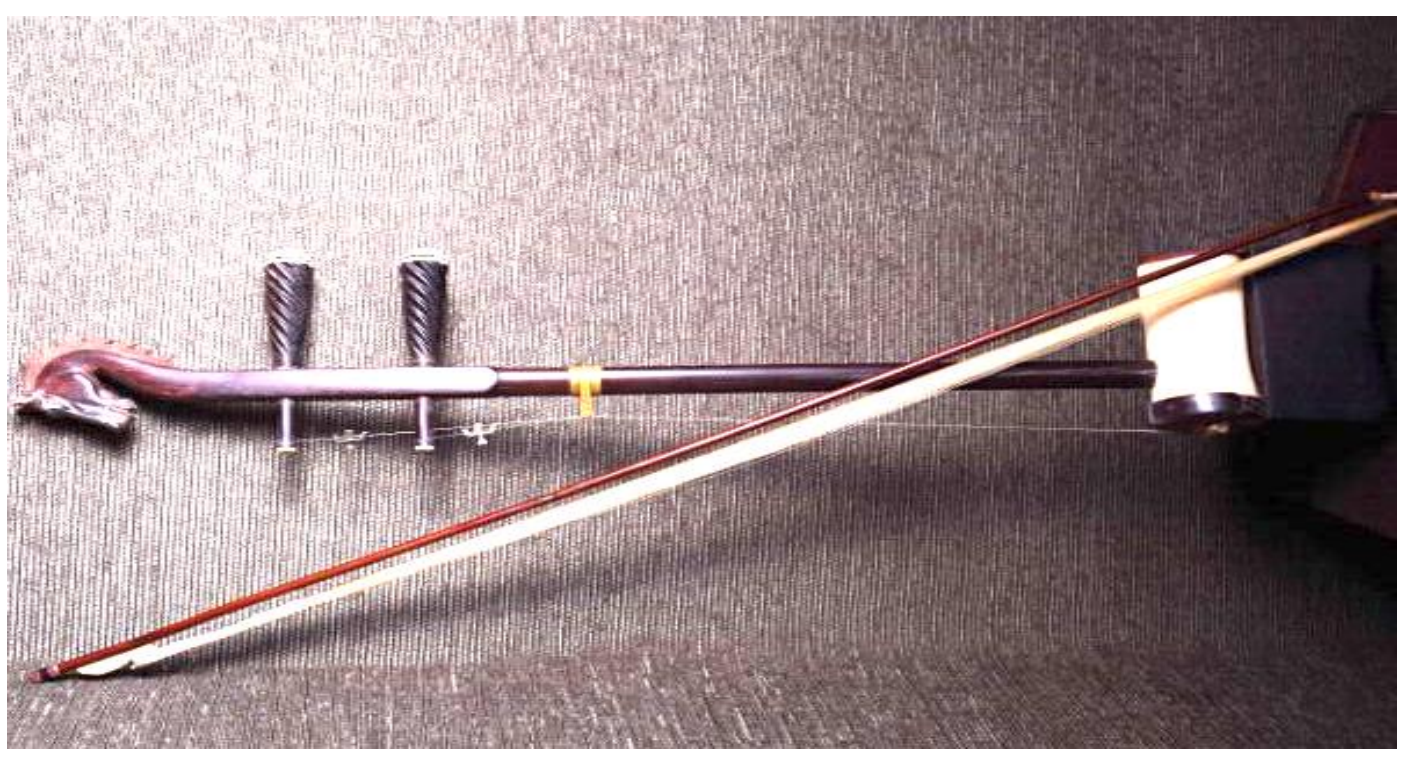

Figure 1. Maguhu, two-stringed bowed fiddle 
Source: https://www.baidu.com/Maguhu, retrieved on January 16, 2019.

In May 2006, Zhuang Opera was embodied in the national intangible cultural heritage preservation list. Tianlin County has held a Zhuang Opera art festival since 2011. With the support of both national policies and the masses, Zhuang Opera has further prospered and developed, has established a foothold in modern society, and has ushered in a new climax of development (Han Wanhong, 2017).

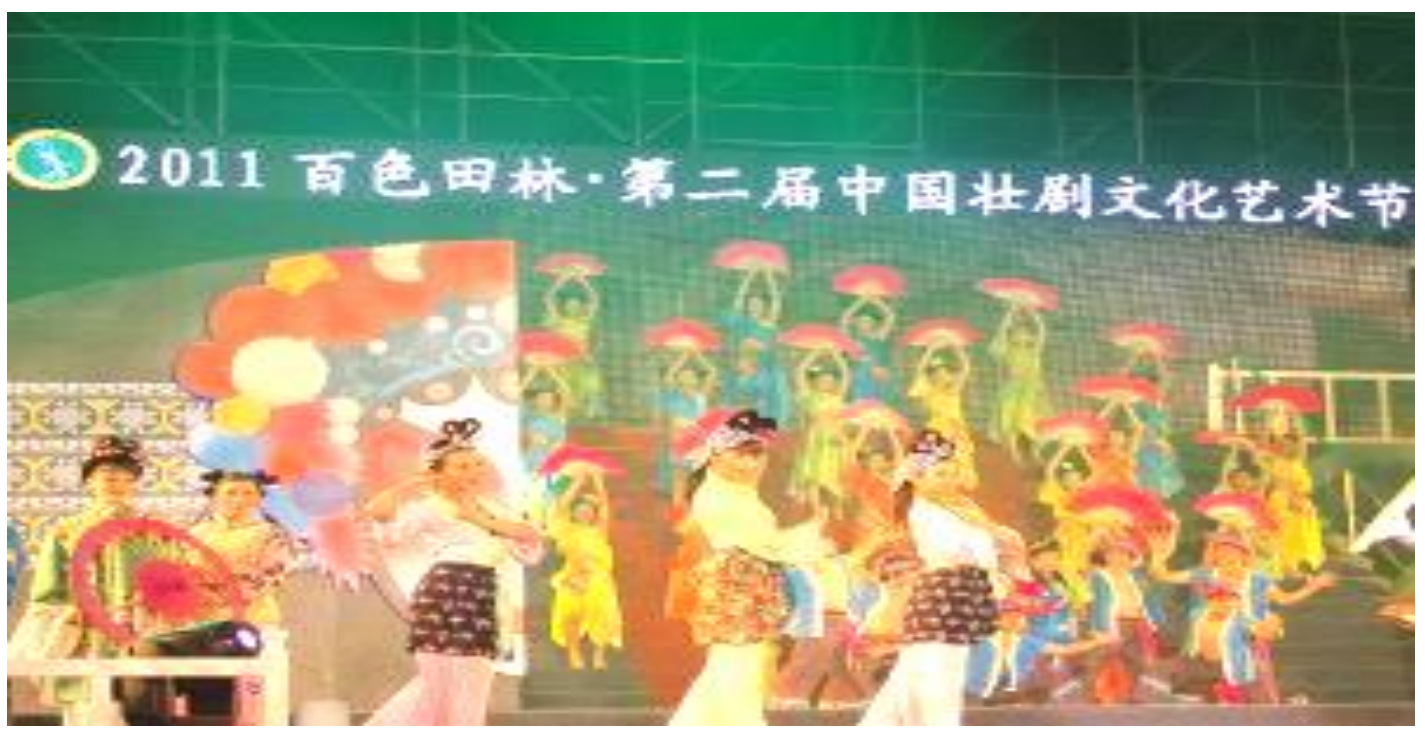

Figure 2. Zhuang Opera Culture and Art Festival 2011

Source: http://gx.people.com.cn/n2/2018/0517/c179430-31593755.html, retrieved on January $16,2019$.

\section{The earliest form of Zhuang Opera --- "Taipingchun"}

"Taipingchun," which means "Peace spring," is written in ancient Zhuang characters, using five-character sentences and waist-feet rhyme (will be discussed in Chapter Six). Because of the content, it is a tribute to the peaceful and prosperous scene of the Qing Dynasty emperor Kangxi's succession, hence the "Peace Spring" (Li Qiong. 2016).

"Taipingchun" has no story content, no characterization, and no opera conflict. It cannot regard as an opera in the traditional sense. However, due to the particularity and importance of its historical status, it cannot be excluded from the discussion of Zhuang Opera, and it classified as a separate discussion. Some scholars have carried out a detailed analysis and research on the content of their singing and explored the generation of Zhuang Opera and its costumes, makeup, and characters, which are all essential for studying the history of Zhuang Opera. This is a rare opera relic in the history of Zhuang literature (Huang Shouheng, 2015). 

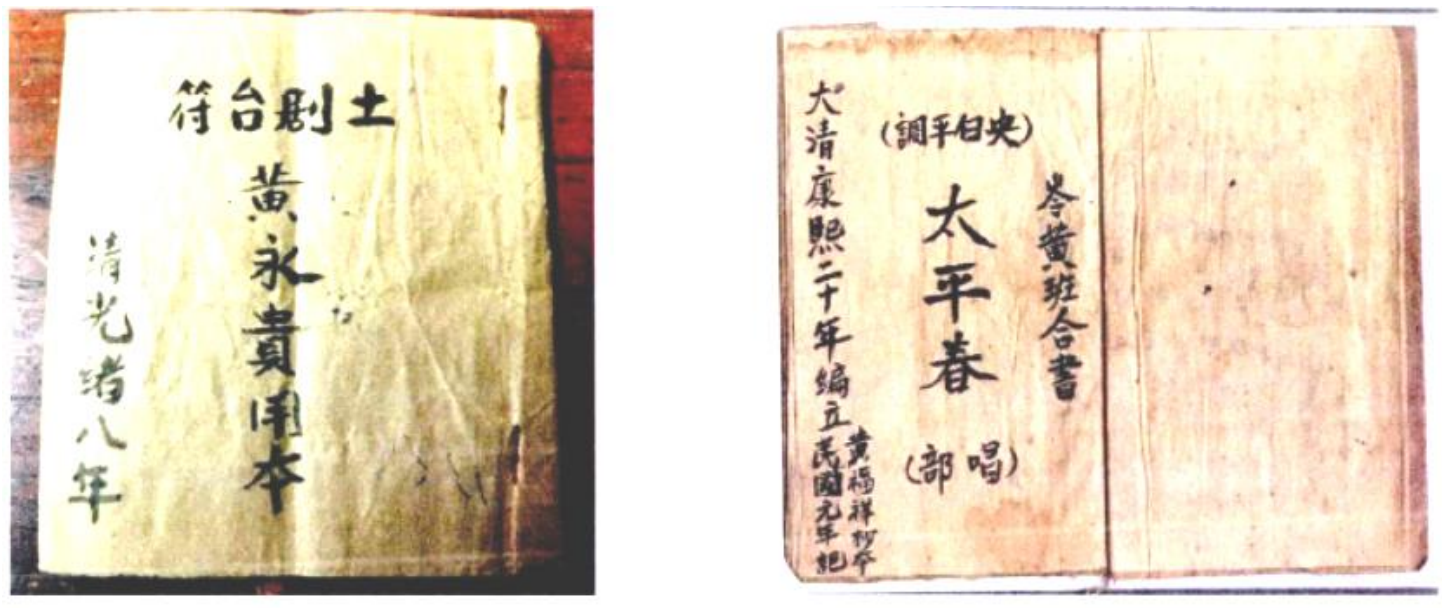

Figure 3. Ancient manuscript of "Tuxi-taifu" and "Taipingchun"

Source: Tianlin County Cultural Museum, photo by Hang Wanhong.

"Taipingchun" was compiled in the 20th year of Kangxi Period in the Qing Dynasty (1681). It is the earliest form of the North Zhuang Opera. It has been transmitted for over 300 years (Ding Shibo, 1995).

It was compiled in the first piece of the "Traditional Chinese Opera Integrated Tianlin Volume," which includes "Beginning Song," "Happy Song," "New House Song," "Baozhai Song," "Zan Baogong," "Zan Shennong," "The Praises of Guanyin," "Praise to the Emperor of the Tang Dynasty," "New Year Song," and "Praise to the Emperor of the Qing Dynasty," each with a length ranging from 16 to 56 sentences, with a total of 308 lines. The content is mainly the singing of various praises and congratulations (Ding Shibo, 1995).

For instance, in the section of "Bao zhai song," it is sung:

English: My village is very beautiful.

It is rich for thousands of years and everything always goes well.

All years are better than the years before, and all generations are happy.

There are banyan trees to protect the villages and longan trees to protect the streets.

Zhuang: $\quad$ Mbanj raeuz ndi lienz ndi, cien bi lij funghyungz.

Miz gorungz bauj mbanj, miz go'nganx bauj gai.

Although there are specific characters in the "Zan Baogong," "Zan Shennong," "Zan Guanyin," "Zan Tang Emperor," and other parts, they also contain praises for the protagonist 
without any storyline. For instance, "Zan Baogong" is translated as follows:

English: $\quad$ In the past, Bao Wenzheng was truly fair.

Sincere to the people, he is a father without officials.

Kindness Bao Wenzheng is a well-known official.

Everyone in Kaifeng is fair.

Zhuang: $\quad$ Mwhnduj Bauh Vwnzcingq, naengh saeq ndaej goengcingq.

Ndij beksingq gangj cin, buxbienga ing lumj boh.

Saemsoh Bauh Vwnzcingq, cin miz mingz cingguen.

Naengh saeq Gaihfunghfuj, buxbux naeuz goengdauh.

Note: The quotes of the opera lines are from the integrated Tianlin Volume of Traditional Chinese Opera Plays, 2013.

This segment content is chanting from "Zan Baogong." Through simple examples of events, it praises Bao Gong's honest character with extremely concise words. The words show the respect of the people. The other chapters contain similar tribute and congratulations without repeated examples.

\section{The music characteristics of Zhuang Opera}

\subsection{The original musical form of Zhuang Opera}

The music of Zhuang Opera developed based on Zhuang folk songs. The evolution of the Ping ban melody of Zhuang Opera can verify this feature. Formerly, in distant history, the Zhuang people chanted the "Song of the Witch" when they prayed to the gods of indigenous religion. Later, the Zhuang women used it to create a song named "Birth of the Spirit," and then some artists adapted it into a new melody --- the Molun-diao melody. This melody is originally from a kind of ritual music of the Zhuang, meaning "Spirit Tale of Storytelling." Then, the Molun melody developed into the main music of the Zhuang Opera named the Ping ban melody (Gan Ling, 2008). More details of the evolutionary process will be analyzed in the following section.

The transcription of the Molun-diao melody is as follows: 


\section{Mo Lun}

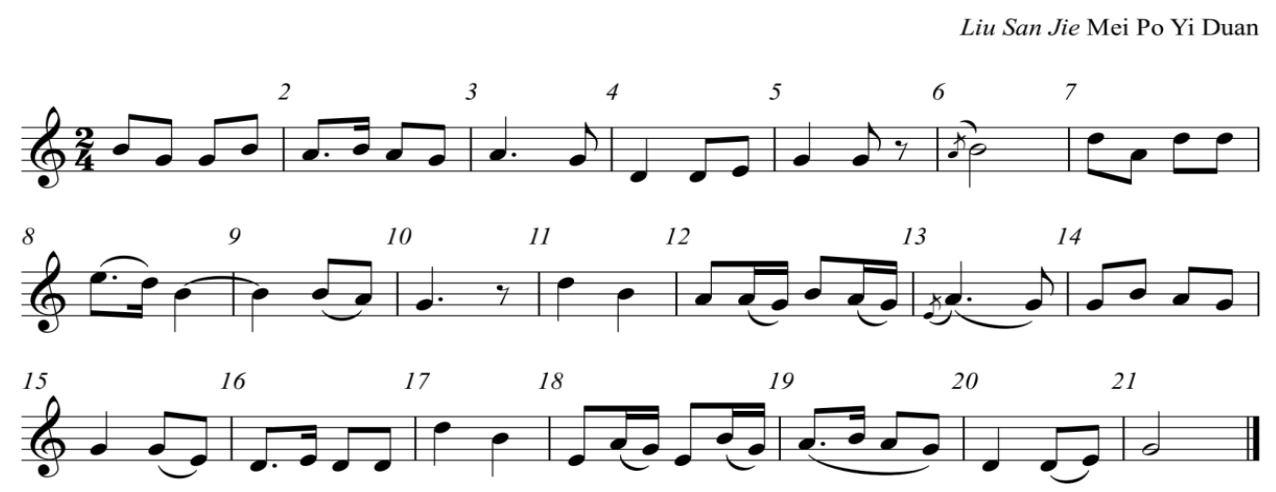

Figure 4. The transcription of the Molun-diao melody

The Ping ban melody developed based on Molun-diao melody. It has evolved into various modes to express the characters' feelings of happiness, anger, sorrow, and excitement. There are 13 melodies mainly used in Zhuang Opera, and four types of Ping ban melody which are divided into different types according to the tempo. Among them are, the Ping ban melody, Tandiao melody, and Ma'ai-pingban melody belonging to the adagio; the Caihua-diao melody and Xidiao melody, which belong to the andante; the Gaoqiang melody and Kuaixi-diao melody belonging to the allegro; and the Shidiao melody, Kudiao melody, and Handiao melody which belong to introduction.

The transcription of the Ping ban melody is as follows:

Ping Ban

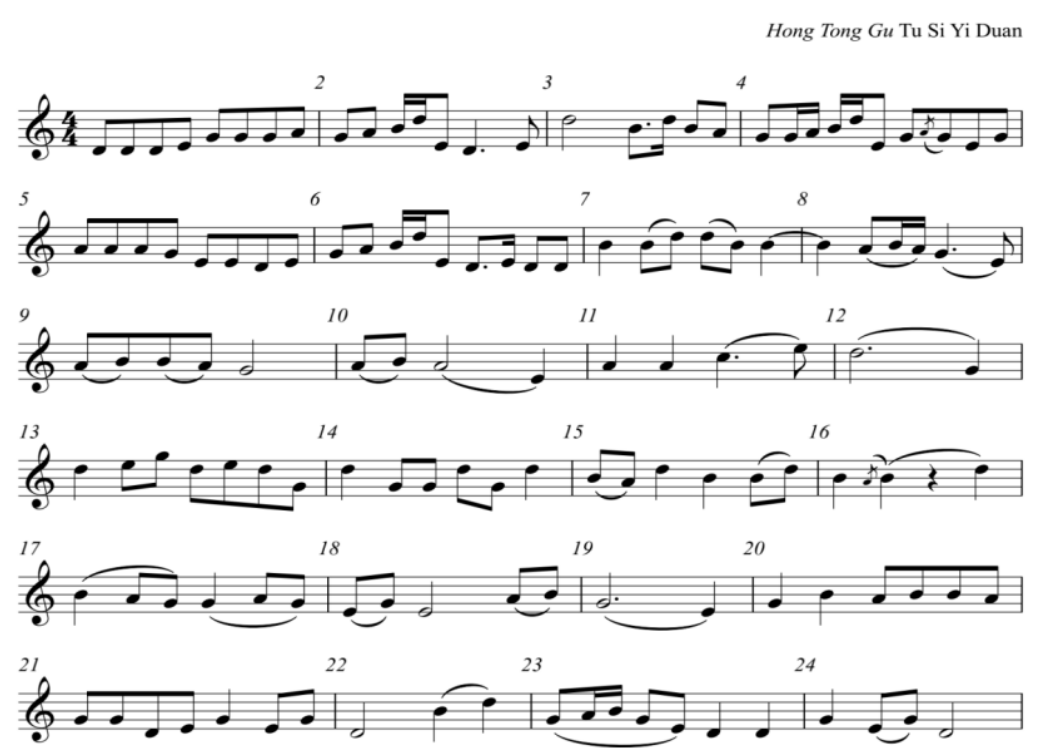

Figure 5. The transcription of the Ping ban melody 
The music structure of the Ping ban melody can be divided into three parts (ternary form A-B-A): the introduction (bars 1-6), the main melody part (bars 7-12), and the ending part (bars 13-24). The register of this melody is: D-E-G-A-B, according to the major third interval (G-B), in which one can identify the gong tone which is G; therefore this melody is in the D zhi mode pattern.

\subsection{Evolution of music in Zhuang Opera}

As mentioned before, the Ping ban melody is the original musical material in the Zhuang Opera, and other melodies are mostly developed based on it.

The evolution of Zhuang Opera music divides two ways: direct relationship and indirect relationship. Among them, the Ping ban melody $\rightarrow$ Ma'ai ping ban melody and Tan diao melody are developed directly. The Ping ban melody $\rightarrow$ Picking Flower melody, Xidiao melody $\rightarrow$ Gaoqiang melody, Kuaixi-diao melody $\rightarrow$ Kudiao melody, Handiao melody, Shidiao melody, Saoban melody, and Sigan melody belong to the indirect relationship category (Edition Committee of Chinese Traditional Operas, 1995). The analysis chart is as below.

Table 2 Original melodies in Zhuang Opera and their variations

\begin{tabular}{ccc}
\hline \hline Original melody & Variation & Relationship \\
\hline Ping ban & Tandiao & \\
Ping ban & Ping ban-neichang & Direct adaptation \\
Picking-flower, Xidiao & Indirect adaptation \\
Xicking-flower & Gaoqiang & Direct adaptation \\
Gaoqiang & Kuaixi-diao & \\
Kuaixi-diao & Kudiao, Handiao & Direct adaptation \\
\hline
\end{tabular}

\section{3 "Yaojiaoyun": The rhyme patterns of Zhuang Opera}

The scripts of traditional Zhuang Operas are all poetic rhythms of Zhuang folk songs. Especially, the South Zhuang Opera Pingban melody and Tandiao melody use Yaojiaoyun (腰 脚韵) rhyme patterns (Chen Wenying, 2014). The Waist-foot pattern refers to the middle (waist) and end (foot) position in a single line of the script text.

In Chinese, "Yao" means waist, "Jiao" in Chinese means feet, and "Yun" means rhythm, so that one can call it in English a "Waist-foot rhyme" pattern. The Waist-foot rhyme pattern is: the last character (foot) of the first phrase which is rhymed with the fifth or third character (waist) of the second phrase (seven phrases are used for the fifth character, and five phrases are used for the third character), the last word of the third phrase rhymes with the fifth or third 
word of the fourth phrase (Han Wanhong, 2017).

For instance, this is used in the third movement of the Zhuang Opera, "Bao Hulu." The transliteration of the sung words of the actor Jiao Da from the Zhuang dialect was selected as an example:

Line1: Mw kai fwn de aeu gang gya (gai)

Line2: Aet bak ha Cen mabah (mai) yen kai (gai)

Line3: De Laih raeu nyaen tai (dai) meu bau (pao)

Line4: Dang dang ndah vam kauh (kao) gang kaen (ga)

Line5: Yeuh boi ha bak haig (ha) ying ngaen

The structure of the Waist-foot rhyme pattern enriches the traditional Zhuang Opera. Simultaneously, in Zhuang Opera vocal performance, ornaments are also one of the commonly used techniques. There are types of actor's lines such as exclamation, appellation, and ornamentation of various functional forms. According to the needs of different melodies, Zhuang Opera uses actors' lines with various rhyme patterns. The plot content's expression effect in the stage performance is enhanced through the use of different forms of rhyme patterns.

\section{Discussion and Conclusion}

From the perspective of ethnomusicology, this research is conducted with the interdisciplinary approach to analyze Zhuang Opera in Guangxi Province. Through the analysis of the activities of Zhuang Opera, and the interpretation of the art form of Zhuang Opera, this study is a contribution to the knowledge about, including its development process, and the earliest form of repertoire, as well as the musical characteristics of Zhuang Opera

Taking three different types of Zhuang Operas in Guangxi as the starting point, this dissertation examines the artistic characteristics, elements of music, and the transmission and development of traditional operas under different times.

In terms of music analysis, the melodic characteristics are analyzed using traditional Chinese and western music theories, while the elements of Zhuang music are analyzed. At the same time, by describing the ritual activities and the performance practices of the Zhuang Opera's, its artistic forms were analyzed. The cross-cultural study of the history and geography of South Zhuang Opera, Shigong Zhuang Opera, and the North Zhuang Opera reveals the factors of the development of the three forms, and their integration into the culture. 


\subsection{Significance of Research}

After more than two hundred years of development, Zhuang Opera has its beautiful vocal music, diverse performances, and rich cultural symbolism. However, even if having been designated as a national intangible cultural heritage in 2006, the current situation of Zhuang Opera is still problematic. Moreover, the practitioners are required to create new styles to express contemporary aesthetics. There are still many aspects that necessitate improvement and study as this is also the audience's higher demand on modern society practitioners.

In terms of concept, this study expands the application of invention of tradition and gives a new explanation to the specific content of the concept. On the other hand, this study can provide reference for researchers who also use this concept. This research can be used as a model of this kind of research to provide guidance for other research topics.

\subsection{Limitations and Future Research Direction}

This study describes the traditional art of Chinese minority in Guangxi Province, and analyzes the phenomenon shown in it. However, there are still a few limitations in this study.

As with any research study, certain factors remain beyond the control of the researcher. With any fieldwork survey, there is always the possibility that inaccurate information may be represented or that individuals may be overstating or understating the facts known to them. The possibility also exists that the individual responding to a question may not be the most knowledgeable person to participate in the institution's survey, or the timing is not quite suitable. Therefore, in future study, attention should be paid more to the role of different groups and experts.

\section{REFERENCES}

[1]. Blacking, John. (1971). Deep and Surface Structures in Venda Music, Yearbook of the International Folk Music Council 3:91-108.1973. How Musical is Man? Seattle: University of Washington Press.

[2]. Bodomo, Adams. (2007). Music and Language in the Strophic Singing of the Zhuang Minority in Southern China. Doctoral dissertation, university of California.

[3]. Becker, Judith. (2004). The relationship of text and melody in Chinese opera. In: Sundberg J., Nord L., Carlson R. (eds) Music, Language, Speech and Brain. Wenner-Gren Center International Symposium Series. Palgrave, London.

[4]. Chen Liqin. (2011). Zhuang Opera and Folk Culture. Journal of Guangxi University for Nationalities (Philosophy and Social Sciences Edition).

[5]. Ding Shibo. (1995). "Tai Ping Chun" is a sing for the embryonic form of Zhuang Opera. National art, 1995(2): 121-128.

[6]. Fang Hechun. (2007). The Collection of Chinese Minority opera Research, Shenyang: Suining National Publishing House.

[7]. Gan Ling. (2008). A Research in the Traditions of Shi Wizard and Folk Memorial Opera in Countryside in Zhuang Nationality. MA Thesis, Guangxi Normal University. 
[8]. Huang Shouheng. (2015). 'Taipingchun’ transmission Information Research. Journal of Wenshan College.

[9]. Huang Shouheng. (2015). 'Taipingchun' transmission Information Research. Journal of Wenshan College.

[10]. He Cui. (2015). Research on Narrative of Chinese Classical opera. MA Thesis, Nanjing University.

[11]. Huang Youxia. (2008). Analysis of the Folklore of Zhuang Opera. Journal of Guangxi University Philosophy and Social Sciences Edition.

[12]. Han Wanhong. (2017). Research on traditional repertoire of North Zhuang Opera. MA Thesis, Minzu University of China.

[13]. Li Fuqiang. (1999). Zhuang Traditional Culture in the Perspective of Anthropology. Nanning: Guangxi People's Publishing House.

[14]. Liao Mingjun. (2008). The Preservation of Zhuang Opera Art and Intangible Cultural Heritage, Nanning: Guangxi People's Publishing House.

[15]. Li Qiong. (2016). On the Transition of Guangxi Beilu Zhuang Opera from Rap to Acting --- Taking "Taipingchun" as a case study. journal of Wenshan university. Vol. 29. No. 2.Apr.

[16]. Lu Guomin. (2008). The Development of Zhuang Opera and the Formation of Music. Art journal.

[17]. Seeger, Charles. (1977). The Musicological Juncture: 1976. Ethnomusicology 21(2):179-188.

[18]. Tian Qing. (2017). How to Avoid the homogenization of minority opera development. People's Political Consultative Conference.

[19]. Widman, John Harvey. (2019). Music and Language in the Strophic Singing of the Zhuang Minority in Southern China. Doctoral dissertation, university of California.

[20]. Wei Wei, Xiang Fan. (1990). Zhuang Opera Art Research, Nanning: Guangxi People's Publishing House.

[21]. Yang Danhua. (2013). Analysis of the Essentials of Zhuang Opera Singing, Art Technology.

[22]. Zhang Jiannian. (1987). Discuss Zhuang Opera. National Art. 